criticisms from Frances and Spitzer, members of the $D S M-V$ task force accused them of protesting the current process for financial reasons: "Both Dr. Frances and Dr. Spitzer ... continue to receive royalties on $D S M-I V$ associated products" (www.psychiatrictimes .com/display/article/10168/1425806? verify $=0$ ).

Some critics of the DSM process express other concerns in addition to matters of transparency. It's been pointed out that about $70 \%$ of current task force members have ties to the pharmaceutical industry, up about $14 \%$ for DSM-IV. A study of an earlier edition of the manual found that ties to the drug industry are particularly strong in working groups focusing on diagnostic areas in which drugs are the first line of treatment (Pscyhother Psychosom 2006;75:154-60). For DSM-IV, all of the members of the working groups for mood disorders and "schizophrenia and other psychotic disorders" had ties to drug companies.

"We recommended that they limit the number of people on these working groups with industry ties, making them a minority so they won't dominate," says Sheldon Krimsky, a coauthor of the study and an adjunct professor in the Department of Public Health and Family Medicine at the Tufts School of Medicine in Medford, Massachusetts. "But that hasn't happened yet."

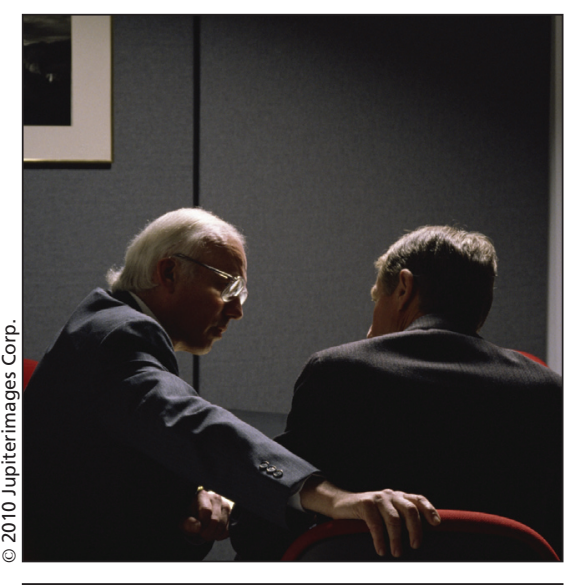

Concerns have been raised that the current revision of the Diagnostic and Statistical Manual of Mental Disorders is too secretive.

The study noted that the pharmaceutical industry funds conventions and research related to disorders proposed for entry in the DSM because "what is considered diagnosable directly impacts the sale of their drugs."

Members of DSM working groups are also wooed by drug companies, Krimsky says, because their involvement with the prestigious manual makes them valuable on the lecture circuit.

"If they start out not having industry connections, they will be tempted by industry to create them," he adds.

But the DSM-V task force claims to be addressing these problems with con- flicts of interest disclosure. Psychiatrists working on the manual are limited to US\$10 000 in consulting fees from drug companies. Defenders of the process also claim that relationships with industry aren't inherently harmful, and that collaboration by government, academia and industry aids development of pharmacological treatments (www.psychiatric times.com/display/article/10168/1364672 ?pageNumber=2).

Another criticism of the current task force is that they are being too ambitious, suggesting that $D S M-V$ will be a "paradigm shift" in psychiatric diagnosis. Frances says a conservative approach to revising the manual is more appropriate. A radical change could only be justified if there were a fundamental leap in the understanding of what causes mental disorders, he says, and though advances in neuroscience and brain imaging show promise, that leap has yet to occur. Too many changes to the DSM will only lead to many people being mistakenly labelled as mentally ill and put on medications without good reason. In his email, Frances says the "ambition to be innovative, when no substantial innovation is possible, will likely lead to arbitrary changes that will often do more harm than good." - Roger Collier, CMAJ

DOI:10.1503/cmaj.109-3108

\title{
Care or killing?
}

Previously published at www.cmaj.ca

$\mathrm{I}$ t's been lauded as an evidence-based framework for delivering appropriate end-of-life or palliative care to the terminally ill, and slagged a self-fulfilling proposition that should be known as the "Liverpool Death Pathway," rather than the Liverpool Care Pathway.

A recent care audit given to dying patients in British hospitals, though, has reviewed the medical protocol favourably (www.liv.ac.uk/mcpcil/liverpool-care -pathway/Generic_NCDAH_2nd_Round _Final_PRINTABLE.pdf).

The controversial pathway, which was modelled on care provided at
United Kingdom hospices for the terminally ill, was initially developed by the Royal Liverpool Hospital and the Marie Curie Cancer Care, a charity providing home or hospice care to terminally ill patients. Although initially developed for cancer patients, the program was later adapted for other conditions.

The pathway is meant to be applied during a dying patient's last few days and hours. It offers guidance in such areas as symptom control, comfort measures, the discontinuation of inappropriate measures and anticipatory prescribing of medication, along with psychological and spiritual care of the patient and family.

"The Liverpool Care Pathway made physicians aware that the diagnosis of dying has consequences for what they did for patients," says Dr. Bill Noble, president of the Association for Palliative Medicine of Great Britain. "Before this, there was a tendency to simply carry on existing treatments until the patient could no longer endure them."

According to a hospital use template, the pathway should be used when "all possible reversible causes" for a patient's condition have been considered, and a multiprofessional team has agreed not only that the patient is dying but that at least two of the following four criteria are present: the patient is bedbound, only able to take sips of fluids, semi-comatose 
or no longer able to take tablets. The patient's condition is reassessed every four hours, with consideration given to comfort, pain management and relief of distressing symptoms.

Noble says the pathway is "not a oneway street," and in cases where patients' conditions do not continue to deteriorate, it is common practice to take them off the pathway and resume previous treatment.

"Every patient is different," Noble says. He cautions that appropriate training and support for health care providers is a crucial part of the pathway's use but that's been patchy, as local National Health Service trusts have discretion over budgetary expenditures. Pathway training involves seminars, as well as advice from a facilitator.

The national audit that gave the pathway a favourable review was the second in two years conducted by the Marie Curie Palliative Care Institute and the Clinical Standards Department of the Royal College of Physicians, supported by the Marie Curie Cancer Care charity and the Department of Health End of Life Care Programme. The audit surveyed pathway use in 155 hospitals and examined the records of almost 4000 patients. Of hospitals surveyed, $74 \%$ said they had continuing education programs for doctors and $84 \%$ for nurses. But only $39 \%$ of hospitals had a facilitator to help staff implement the program.

The audit also found that the vast majority of patients were reported to be comfortable during their last 24 hours, with $65 \%$ needing no subcutaneous infusion of medication to control discomfort, $31 \%$ receiving low doses of subcutaneous medication and only $4 \%$ requiring high doses.

The audit also indicated that approximately three-quarters of patients' families or family caregivers were advised of the plan, and that communication between them and hospital staff could be improved. As well, it faulted the provision of spiritual and religious care, noting that assessment of these needs was achieved in only $30 \%$ of patients and just $50 \%$ of their families.

Despite the positive review, the pathway is increasingly being criticized as more hospitals adopt the program. Recently, a group of six medical experts wrote in a letter to the Daily Telegraph

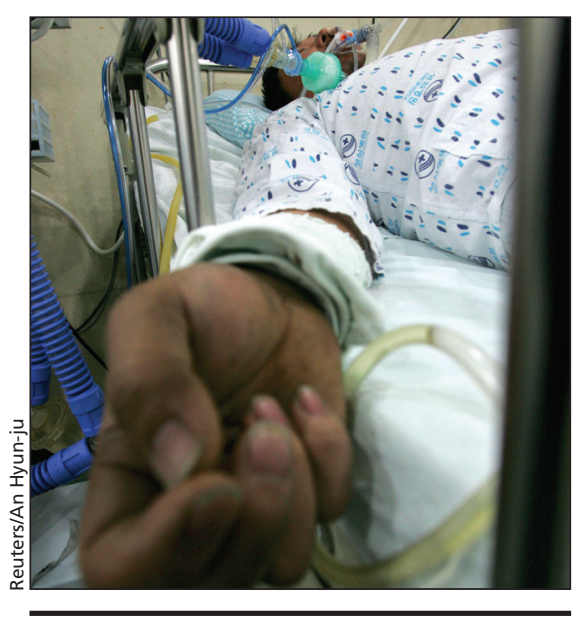

It is recommended that the Liverpool Care Pathway be used when "all possible reversible" causes for a patient's condition have been considered and a multiprofessional team agrees that at least two of four conditions are present: the patient is bedbound; only able to take sips of fluids; is semi-comatose; or no longer able to take tablets.

that forecasting death is an inexact science. "The government is rolling out a new treatment pattern of palliative care into hospitals, nursing and residential homes. It is based on experience in a Liverpool hospice. If you tick all the right boxes in the Liverpool Care Pathway, the inevitable outcome of the consequent treatment is death."

One of those six letter writers, Dr. Anthony Cole, chairman of the Medical Ethics Alliance, says the pathway "is quite flexible, with checks and balances built into it," but that a lack of adequate training has resulted in its misuse, sometimes with lethal effects.

"It is important that patients know they can have a second opinion," he says.

Somewhat surprisingly, the British anti-euthanasia group, Care Not Killing, supports the pathway. It recently issued a press release stating that the pathway has a value "in providing a framework for decision-making by doctors treating imminently dying patients."

"Unlike practices in other countries, such as the Netherlands where deep continuous sedation until death is administered according to a protocol, palliative care physicians in Britain have the skills to ensure that the overwhelming majority of terminally ill patients are able to die peacefully and without any significant sedation," the antieuthanasia group added.

Critics have included relatives of elderly patients who may have been inappropriately placed on the pathway.

Michael Danby, a solicitor of the family of Jack Jones, an elderly cancer survivor who was incorrectly informed that the disease had returned and sent to a Marie Curie hospice, where he died, says that no tests were carried out to verify whether Jones' cancer actually returned.

A postmortem revealed no cancer recurrence and that Jones had in fact died of pneumonia, an illness for which he received no treatment while at the hospice, Danby says. Jones' widow received an $£ 18000$ out-of-court settlement, but the hospice and attending physicians have denied liability in the case.

Similarly, the daughter of 80-yearold Hazel Fenton says that her mother was suffering from pneumonia when she was admitted to the Conquest Hospital in East Sussex, where she was told she was dying and placed on the pathway. A nurse even asked what the woman wanted done with her mother's body. It took several days to persuade hospital staff to provide artificial feeding to Fenton, who was later moved to a nursing home and is alive nine months later.

It indicates that the pathway should be used with caution, says Dr. Sam Ahmedzai, professor of palliative medicine at the University of Sheffield.

"The Liverpool Care Pathway is for the last one to three days of a patient's life, and if more time elapses they need to be taken off the plan," says Ahmedzai. "The difficulty is when the LCP is applied in settings where the staff lack the training, are very busy or short-staffed. Patients need to be continually assessed, and in busy wards that doesn't always happen."

Ahmedzai also notes that while cancer patients have "traditionally got the better deal" in palliative care, the pathway has resulted in improved end-of-life care for patients with other conditions. "There are pathways, or protocols, for all conditions and we never want to go back to the preprotocol days," he adds. - Mary Helen Spooner, London, England

DOI:10.1503/cmaj.109-3104 